\title{
Propuesta de enfermería con terapias complementarias para el abordaje de riesgos psicosociales a nivel laboral $^{1}$
}

\author{
ENSAYO
}

\author{
Paula Andrea Ceballos Vásquez ${ }^{2}$ \\ Vivian Vílchez-Barboza ${ }^{3}$ \\ Sandra Valenzuela-Suazo ${ }^{4}$
}

Institución: Universidad Católica del Maule

COMO CITAR

Ceballos, P.A., Vílchez-Barboza, V. y Valenzuela-Suazo, S. (2016). Propuesta de enfermería con terapias complementarias para el abordaje de riesgos psicosociales a nivel laboral. Rev. Enfermería Actual en Costa Rica, 30, 1-13. DOI: http://dx.doi.org/10.15517/revenf.v0i30.22206

\section{RESUMEN}

Introducción. Es reconocido que el trabajo genera efectos positivos en las personas como seguridad económica, bienestar y satisfacción, aspectos que ofrecen beneficios en todo ámbito, inclusive en la salud. A pesar de lo anterior, el mundo laboral en la actualidad exige tomar decisiones, pensar críticamente, liderar, además de poseer conocimientos para ejecutar las labores en forma eficiente y eficaz, lo cual puede producir sobrecarga y disconfort que afectan física, psicológica y socialmente a un individuo.

Desarrollo. El propósito de este trabajo es aportar al enfrentamiento de los riesgos psicosociales en el ámbito laboral, por medio de la utilización de terapias complementarias y/o alternativas desde la disciplina de Enfermería. Se postula que la enfermería posee un campo de saberes, ligados al cuidado holístico y a la promoción en salud, por ello se toma el marco legal actual y las bases disciplinares de Enfermería, las cuales dan soporte a la implementación de terapias complementarias o alternativas en salud laboral.

Conclusión. Como consideraciones finales, se destaca que las bases disciplinares de la enfermería abogan por el cuidado de los trabajadores a través del uso de terapias complementaria, de modo que otorgue fundamentos teóricos y científicos al área de salud laboral para enfrentar los riesgos psicosociales en el trabajo desde una mirada trasformadora.

Palabras clave: Enfermería, riesgos, terapias-complementarias, trabajo, salud laboral

\footnotetext{
${ }^{1}$ Fecha de recepción: 25 de setiembre del 2015

Fecha de aceptación: 20 noviembre del 2015

${ }^{2}$ Enfermera. Académica Departamento de Enfermería. Universidad Católica del Maule. Chile. Correo electrónico: pcpaulaandrea6@gmail.com

${ }^{3}$ Enfermera. Profesora Escuela de Enfermería. Universidad de Costa Rica. Costa Rica. Correo electrónico: vivian.vilchez@ucr.ac.cr

${ }^{4}$ Enfermera. Facultad de Enfermería. Universidad de Concepción. Chile. Correo electrónico: fvalenzu@udec.cl
} 


\title{
Proposal to nursing with complementary therapies for the approach psychosocial risk factors at work ${ }^{1}$
}

\author{
ESSAY
}

Institution: Catholic University of Maule

\author{
Paula Andrea Ceballos Vásquez ${ }^{2}$ \\ Vivian Vílchez-Barboza ${ }^{3}$ \\ Sandra Valenzuela-Suazo ${ }^{4}$
}

\section{CITED:}

Ceballos, P.A., Vílchez-Barboza, V. y Valenzuela-Suazo, S. (2016). Proposal to nursing with complementary therapies for the approach psychosocial risk factors at work. Rev. Enfermería Actual en Costa Rica, 30, 1-13. DOI: http://dx.doi.org/10.15517/revenf.v0i30.22206

\begin{abstract}
Introduction: It is recognized that the work generates positive effects on people and economic security, wellbeing and satisfaction, both of which offer benefits in all areas, including health. Despite this, the world of work now requires decisions, think critically, lead, besides having expertise to perform tasks efficiently and effectively, which may result in overload and discomfort affecting physically, psychologically and socially an individual.

Developing: This article is a theoretical reflection conducted with the purpose of contributing from the discipline of nursing, facing the psychosocial risk factors at work through the usage of complementary and/or alternative therapies. This is proposed since nursing possesses knowledge linked to holistic care and healthcare promotion. For that purpose, the current legal framework globally and nationally, and the disciplinary bases for nursing, are presented, which contribute to the implementation of complementary or alternative therapies on occupational health.

Conclusion: As closing remarks, it is proposed that nursing possesses disciplinary bases which advocate for the workers healthcare through the usage of complementary therapies. Therefore, that profession might present theoretical and scientific fundamentals to the occupational healthcare in order to face psychosocial risk factors at work with a transforming perspective.
\end{abstract}

Key words: Nursing, Risks, Complementary-Therapies, Work, Occupational-Health

\footnotetext{
${ }_{2}^{1}$ Date of receipt: September 25, 2015

Date of acceptance: November 20, 2015

${ }^{2}$ Nurse. Department of Nursing Academic. Catholic University of Maule. Chile. Email: pcpaulaandrea6@gmail.com

${ }^{3}$ Nurse. Professor at the University of Costa Rica. Costa Rica. Email: vivian.vilchez@ucr.ac.cr

${ }^{4}$ Nurse. School of Nursing. University of Concepción. Chile. Email: fvalenzu@udec.cl
} 


\section{Revista Electrónica Enfermeria Actual en costa Rica}

\section{INTRODUCCIÓN}

Es reconocido que el trabajo genera efectos positivos en las personas como seguridad económica, bienestar y satisfacción, aspectos que ofrecen beneficios en todo ámbito, inclusive en la salud. A pesar de lo anterior, el mundo laboral en la actualidad exige tomar decisiones, pensar críticamente, liderar, además de poseer conocimientos para ejecutar las labores en forma eficiente y eficaz, lo cual puede producir sobrecarga y disconfort que afectan física, psicológica y socialmente a un individuo. Autores afirman que "el ritmo de vida de la sociedad occidental ha generado el aumento de estrés laboral y de los problemas asociados a él” (Gil-Monte, 2005, p.19).

Sin lugar a dudas, lo mencionado constituye una fuente de riesgos laborales y problemas de salud en todas sus dimensiones. En lo que respecta a lo psicológico o mental, los problemas se relacionan con la sobrecarga de trabajo, la baja utilización de habilidades y otros factores ocupacionales que implican presión laboral, conflicto y ambigüedad en las funciones (Hernández, Terán, Navarrete y León, 2007). Tales riesgos son conocidos como psicosociales e incluyen de manera indistinta elementos externos a la persona, llamados exigencias laborales, tales como las cargas de trabajo, demandas y estresores a las que son sometidos los trabajadores, así como los efectos o respuestas que se generan en ellos (Martínez y Hernández, 2005), como el estrés, el cual puede afectar seriamente el logro de los objetivos de un sector o la totalidad de la institución y, cuyo buen manejo contribuye al éxito organizacional (Panizzon, Luz y Fensterseifer, 2008).

Otra alteración es el síndrome de burnout o de "estar quemado" por el trabajo que se constituye en uno de los daños laborales de carácter psicosocial más importante en la sociedad actual, al punto de que se considera que su abordaje es una necesidad social orientada a mejorar la salud y calidad de vida de las personas (Salanova y Llorens, 2008).

Por otra parte, la ejecución de una o varias tareas puede llevar a los trabajadores/as a percibir que su labor produce sobrecarga mental de trabajo, divididas en cargas fisiológicas, derivadas de esfuerzos visuales y físicos necesarios en la actividad, y psíquicas, relacionadas con la tensión que genera el trabajo, consecuencia del ritmo y la intensidad con la que se ejecuta las tareas, la convivencia con actividades de alta carga emocional, la necesidad de mantenerse actualizado, las condiciones competitivas del mercado, entre otros (Secco et al, 2010).

Actualmente, para enfrentar dichas problemáticas, las estrategias utilizadas por la salud ocupacional se visualiza desde lo patocéntrico, por ende, si el trabajador no presenta alteraciones medibles desde lo biológico, no es valorado, evaluado, ni atendido. Tal situación invita a que la enfermería, desde sus bases disciplinares, brinde sustento teórico y metodológico en el abordaje de estos riesgos, incorporando el uso de terapias complementarias o alternativas para el cuidado de las personas.

En relación con lo anterior, según plantea Sobrin (2009), la salud forma parte de un concepto amplio por lo que la utilización de estas terapias como complemento al sistema actual puede ser muy útil en problemas emergentes en la sociedad, los cuales son producto de los estilos de vida que condicionan la aparición de enfermedades y malestares que se vinculan -fundamentalmente- con elevadas demandas y baja capacidad de adaptación. Al respecto, se plantea que las terapias complementarias son abordajes que objetivan la asistencia a la salud de individuo, del que consideran su mente, cuerpo y espíritu y al que visualizan como un todo y no como un conjunto de partes aisladas (Ceolin, Heck, Pereira, Martins, Coimbra y Silveira, 2009). 
En torno al tema, algunos investigadores sugieren que la expansión de las perspectivas sobre las medicinas complementarias permitirá al profesional en enfermería adquirir mayores conocimientos sobre los cuidados que realizan a personas en distintas culturas en todo el mundo, objetivo trascendental para lograr un cuidado de salud competente (Snyder y Lindsquit, 2011). Dado lo anterior, el propósito de esta reflexión es contribuir al abordaje de los riesgos psicosociales en el trabajo, desde la enfermería, por medio de terapias complementarias.

\section{DESARROLLO}

\section{Factores psicosociales y riesgos psicosociales en el trabajo}

Los factores psicosociales son condiciones presentes en una circunstancia directamente relacionada con la organización del trabajo, su contenido y la realización de la tarea, con capacidad de afectar positiva o negativamente al desarrollo del trabajo, y la salud física, psíquica y/o social del trabajador (Taub y Olivares, 2009). En torno a lo mencionado, el comité mixto OIT/OMS, en la novena reunión sobre medicina del Trabajo, celebrada en Ginebra en 1984 acordó que

los factores psicosociales en el trabajo consisten en interacciones entre el trabajo, su medio ambiente, la satisfacción en el trabajo y las condiciones de su organización, por una parte, y por la otra, las capacidades del trabajador, sus necesidades, su cultura y su situación personal, pueden influir en la salud y en el rendimiento y la satisfacción en el trabajo (OIT/OMS, 1984).

La evidencia ha demostrado que tales factores pueden ser elementos positivos que producen bienestar en los trabajadores, mas también podrían constituirse en elementos negativos que se trasforman en un riesgo para la salud hasta causar ausentismo, desmotivación y bajo desempeño, es decir, cuando los trabajadores los perciben como negativos pasan a ser factores de riesgos, que potencialmente provocarían daños psicológicos, fisiológicos o sociales en las personas (Taub y Olivares, 2009).

Diversos autores indican que los riesgos psicosociales se clasifican en tres grupos relacionados con la organización general del trabajo, con las tareas específicas de cada trabajador y con las interacciones sociales (Meliá et al, 2006; Gil-Monte, 2009; Peiró, 2004), los cuales son agravados con la aparición de riesgos psicosociales emergentes, especialmente en el sector servicios, los cuales pueden afectar la identidad de quien trabaja. Un ejemplo de este nuevo tipo de riesgo es el trabajo emocional, el cual se relaciona con la autorregulación de las emociones, de modo que se inhibe las emociones negativas y se expresa emociones positivas de manera forzada, porque el puesto de trabajo así lo amerita (Moreno, 2011; Nogareda et al, 2004). Tal concepto se vive a diario en los lugares donde se entregan servicios, y un desafío a ser explorado.

\section{¿Cómo se abordan en la actualidad los riesgos psicosociales en el trabajo?}

Desde las metodologías de evaluación de Riesgos Psicosociales, se indica que no basta con la evaluación y valoración de los peligros y daños, sino que esto es solo el inicio (Salanova y Llorens, 2008). Se indica que esto se realiza a nivel general, como intervención de Riesgos Psicosocial, o a nivel específico, implementando estrategias, según la problemática que presente el usuario. 
Por otro lado, expertos señalan que las intervenciones para abordar los riesgos psicosociales se agrupan en tres categorías (Landsbergis, 2008; Martínez, 2010):

-Prevención primaria: se puntualiza en el contexto económico, político y organizativo, de manera que logre cambios sociales, organizativos y de diseño del trabajo.

-Prevención secundaria: se encamina a la respuesta al estrés físiológico, psicológico y de comportamientos, por medio de la detección precoz de alteraciones.

-Prevención terciaria: se encamina al tratamiento y rehabilitación de enfermedades relacionadas con el estrés. Se indica que en cada categoría, las estrategias pueden enfocarse tanto a nivel individual, grupal y/o organizacional. Estas estrategias buscan que los trabajadores tengan una mejor calidad de vida relacionada con salud (CVRS), entendida como la valoración que realiza una persona, de acuerdo con sus propios criterios de estado físico, emocional y social en que se encuentra en un momento dado, además de que refleja el grado de satisfacción con una experiencia personal, a nivel fisiológico, en todas las áreas de su actividad.

En cuanto a la CVRS, contribuye a que las empresas tengan personas saludables y motivadas para la conquista de sus metas y objetivos organizacionales, en donde la interacción persona-empresa sea un espacio socio-temporal, con oportunidades de crecimiento y desarrollo para el colaborador y la organización (Quiceno y Vinaccia, 2007). Con la finalidad de abarcar un mayor número de personas, disminuir costos y mejorar la efectividad de las estrategias, las empresas utilizan intervenciones grupales, uno de los grandes problemas de las propuestas de intervención, especialmente en situaciones de estrés, ya que la percepción de riesgos psicosociales en el trabajo es un fenómeno que ocurre de forma diferenciada para cada individuo (Stacciarini y Tróccoli, 2002). Así, las estrategias grupales o "estandarizadas", serían menos eficientes y eficaces, ya que como se indicó, estas problemáticas son de carácter individual, se vivencian de forma heterogénea, y requieren cuidados diferenciados.

Dada la información anterior, se sugiere la incorporación de otras estrategias que permitan un camino teóricamente sólido, aunque empíricamente limitado, especialmente en la temática de salud laboral latinoamericana, tal como es la sanación con terapias alternativas y/o complementarias.

\section{Una propuesta de abordaje desde enfermería}

El abordaje de los riesgos psicosociales depende principalmente de dos aspectos: el primero, el organizacional, en el cual enfermería tiene un rol más fiscalizador e investigativo y que, al parecer de las autoras (sin desmerecer su importancia), requiere de actores externos, para su abordaje; y el segundo, que se dirige en forma directa al apoyo y/o entrega de herramientas a los trabajadores. Sobre el aspecto mencionado, la enfermería puede desarrollar un campo de saberes, ligados al cuidado holístico, a la promoción de la salud de los usuarios (en este caso trabajadores), con una mirada trasformadora, integral, sanadora y personalizada al implementar el sistema de conocimiento conceptual-teórico-empírico (sistema C-T-E), el cual se define como el servicio que se brinda a la sociedad guiado por el conocimiento específico de la disciplina al articularse con sus teorías (Fawcett, 2005); de manera que otorgue a las personas la posibilidad de ser cuidados según sus requerimientos y significados, con la utilización de terapias complementarias. 
En relación con lo anterior, diversos autores indican que el profesional de enfermería se le demanda la práctica de estas intervenciones, por su enfoque en cada individuo, el cual responde a las necesidades, inquietudes y expectativas particulares, sin olvidar que la búsqueda del "sentirse mejor" se relaciona con elementos no solo físicos, sino emocionales y espirituales (Cabieses, Miner y Villegas, 2010).

Otros expertos puntualizan que -como profesión- la enfermería tiene una base apropiada para comprender la necesidad de reducir el estrés en los usuarios y así favorecer su curación (Pestka y Cutshall, 2011), por lo que la posibilidad de trabajar los riesgos psicosociales presentes en los lugares de trabajo, desde esta mirada, se hace imperiosa y es una oportunidad de desarrollo profesional.

\section{Definición, clasificación y marco normativo de las terapias complementarias}

Un aspecto interesante de dilucidar es el aumento en el uso de las terapias mencionadas por parte de la población; al respecto, se menciona algunas explicaciones como las siguientes (Snyder, Niska y Lindsquit, 2011): a) La filosofía holística implícita en ellas, la cual difiere en grado significativo de la filosofía cartesiana que permea la medicina occidental. Para la aplicación de terapias alternativas, se considera a la persona en su integralidad, debido a que se persigue traer armonía al interior del ser humano.

b) Usuario/a desea ser partícipe en la toma de decisiones respecto de su salud.

c) La calidad de vida, dado que las personas no desean que el tratamiento de su problema de salud sea peor que este, de manera que privilegian la sanación enfocada en la armonía interna.

De esta manera, los profesionales de la salud y la sociedad, deben conocer cómo se definen las terapias complementarias y lo que los organismos internaciones indican al respecto; por ejemplo, la Organización Mundial de la Salud (OMS), en el año 2002 señaló que la medicina tradicional (MT), es un término utilizado para referirse tanto a los sistemas de medicina tradicional como a las diversas formas de medicina indígena, motivo por el que la define como

prácticas, enfoques, conocimientos y creencias sanitarias diversas que incorporan medicinas basadas en plantas, animales y/o minerales, terapias espirituales, técnicas manuales y ejercicios aplicados en forma individual o en combinación para mantener el bienestar, además de tratar, diagnosticar y prevenir las enfermedades (OMS, p.7, 2002).

Como sinónimo del término también se utiliza el de medicina alternativa o medicina complementaria (MCA), aunque según se indica no son lo mismo, así lo afirman distintos expertos que afirman que las MCA corresponden a

un amplio dominio de recursos de sanación que incluye todos los sistemas, modalidades, prácticas de salud, teorías y creencias que los acompañan; diferentes a aquellas intrínsecas al sistema de salud políticamente dominante en una sociedad particular en un período histórico dado (Gobierno de Chile Ministerio de Salud [MINSAL], 2012).

De igual modo, el National Center for Complementary and Alternative Medicine (NCCAM) en el año 2008 definió las MCA, como "un grupo de formado por distintos sistemas médicos y de atención de la salud, 


\section{Revista Electrónica Enfermeria Actual en costa Rica}

prácticas, productos que no se consideran en la actualidad como parte de la medicina convencional" (Snyder et al, 2011, p. 4). Considerando lo mencionado, la gran diferencia entre ambas es que la MT se relaciona con culturas ancestrales, filosofías y actitudes personales y se diferencian según territorio, mientras que la MCA se refiere a una grupo de prácticas sanitarias que no forman parte la tradición de un grupo o de un país pero, están integradas en su sistema sanitario prevaleciente.

El uso de las MT y MCA ha aumentado a nivel mundial, aspecto que llama la atención porque este aumento es mayor en los países desarrollados, por ejemplo, estudios realizados indican que en Australia un 46\% de la población usa MCA; en Francia, un 49\% de la población puntualiza haber utilizado MT y MCA, y en Canadá un $70 \%$. Debido a su auge en el sistema sanitario, la OMS ha incentivado a los gobiernos a reconocer la importancia de la contribución de las MT y MCA, a través de la generación de políticas y normativas nacionales sobre ellas (OMS, 2002).

De acuerdo con lo mencionado, es evidente el respaldo internacional en cuanto al uso estas terapias en alteraciones de salud, como las producidas por los riesgos psicosociales presentes en los lugares de desempeño de los trabajadores: en Chile, en el año 2012 se realizó un estudio en relación con el uso de MAC con una muestra de 498 personas distribuidas en seis ciudades, del cual se destaca que el $55 \%$ ha utilizado MAC, independiente del sexo, edad, nivel socioeconómico e -incluso- del sistema de previsión de salud. Además, el 25,5\% indicó usar este tipo de terapias para enfrentar problemas psicológicos y/o emocionales como el estrés, ansiedad, depresión, entre otros (Gobierno de Chile, 2012).

El número de terapias posibles es cuantioso, debido a que existen más de mil tipos, algunas de ellas muy utilizadas con un gran soporte científico y otras escasamente conocidas, por lo que la NCCAM (2008)(citado por Snyder et al, 2011), las clasificó en las siguientes cuatro categorías.

- En relación con las terapias mente - cuerpo, indica que desde la perspectiva holística, el espíritu se encuentra incluido, pues trata de un aspecto del ser humano. En este grupo se incluyen terapias que favorecen la capacidad de la mente para impactar sobre el funcionamiento del cuerpo (NCCAM, 2008 citado por Snyder et al, 2011 ).

- En cuanto a las terapias con bases biológicas, son las más utilizadas, las cuales incluyen el uso de fitoterapia o uso de hierbas que pueden interaccionar con medicamentos, por lo que es necesario que todos los profesionales de la salud, especialmente enfermería, cuenten con conocimientos al respecto.

- Respecto de las terapias energéticas o del biocampo, son las que utilizan energía originada en o cerca del cuerpo. El concepto de energía y su uso es universal ( $Q i$ en China, Ki en Japón, Prana en India, como ejemplos). Su eficacia es subjetiva, ya que es complejo medir sus efectos en relación con la energía personal (Snyder y Lindsquit, 2011).Cabe destacar que dichas mediciones son absolutamente biomédicas, por lo que se sigue valorando este sistema de terapias con parámetros alopáticos.

\section{Bases disciplinares de enfermería, para su aplicación}

Desde la década del cincuenta, la enfermería propone numerosas filosofías, teorías y modelos, aplicados en la práctica diaria, según lo indican estudios publicados al respecto (Mejías, 2008; Navarro, Castro, 2010). Al 
parecer, no ocurre lo mismo en materia de enfermería en salud laboral, ya que en la revisión bibliográfica no se localizó publicaciones relacionadas con estas temáticas, y al parecer las existentes se vinculan más con la exposición a riesgos psicosociales por parte del colectivo de enfermería, que con la aplicación sus bases teóricas para su abordaje.

Por lo anterior, esta propuesta se basa en plantear que desde los paradigmas de enfermería (Humanbecoming y transformación), se puede sustentar un abordaje holístico para hacer frente a los riesgos psicosociales. Para ello, se postula que, desde Rosemarie Rizzo Parse y Bárbara Dossey, la enfermería podría visibilizar la disciplina y aportar con medidas de mejora en salud ocupacional, debido a que desde el abordaje de dichas enfermeras se visualiza a la persona como un todo, en vez de reducirla a sistemas o partes constituyentes para ser comprendidos (Mitchell, 2007), lo que permite, desde sus saberes, postular un trabajo a nivel psicosocial, orientado a una persona que se encuentra sin patologías, aunque con dolencias o alteraciones que escasamente se evidencian en el sistema biológico, sin olvidar que enfatizan la promoción y prevención de estos riesgos en espacios de trabajo.

En cuanto al paradigma del "Humanbecoming” (Mitchell, 2007), Rosemarie Rizzo Parse (2014) plantea la enfermería como una ciencia, cuya razón principal es el ser humano, al cual llama humanuniverse, un ser indivisible de su ser y del entorno (es la sumatoria de lo visible y lo invisible del ser), por lo que es impredecible y se encuentra en un constante cambio, mediado por procesos ligados con costumbres, relaciones interpersonales, valores, contacto con los diferentes entornos (familiar, laboral, social, entre otros) y los fenómenos que se estructuran a partir de la experiencia de vida (miedo, alegría, esperanza, paz, seguridad). De igual modo, la enfermera mencionada enfatiza que el ser humano toma decisiones y desarrolla capacidades de elección, de acuerdo con la vivencia de sus experiencias de las que construye su realidad personal.

Los supuestos que sustentan este paradigma son: el humanuniverse, como dogma básico, considerando que el ser humano no es una cosa u objeto, sino una unidad con el ser, que cocrea su realidad y es ilimitable en su contexto. El ethos, se refiere a la dignidad y el respeto por el ser humano, a la verdadera presencia que debe tener la/el enfermera/o cuando brinda cuidado. El vivir con calidad, considera que el ser humano está en constante cambio, es un ser que emerge con lo visible e invisible, es lo que otorga su expresión personal única, lo cual enfermería debe considerar al brindar su intervención.

Como parte del abordaje, dicha experta utiliza el prefijo "co" en muchas de sus palabras para denotar la naturaleza participativa de las personas: para ella, "co", significa estar con, lo cual relaciona con el hecho de que los seres humanos no pueden separase nunca de las relaciones con el universo, las cuales incluyen vínculos con los individuos, con la/el enfermera/o, con ideas, proyectos, antecedentes, historia y cultura.

Con el fin de sustentar la aplicación de este paradigma en salud laboral utilizando terapias alternativas, se indica que Parse visualiza al ser humano como un todo, el cual tiene la posibilidad de elegir en forma libre, con intencionalidad y cocrear desde su interior e interelacionalmente su propia mejora, en este caso, con el fin de disminuir los efectos de los riesgos psicosociales en el trabajo, con el propósito de mejorar su calidad de vida.

En cuanto a la Teoría de enfermería integral, desarrollada por Bárbara Dossey $(2008$, 2009), se describe como una cosmovisión integral que incluye teorías holística y otros paradigmas. La autora indica que el 
profesional de enfermería para realizar un proceso de cuidado, debe darse un espacio de exploración interior que le permita la creatividad, la pasión y el amor. En la literatura revisada, se describe esta teoría, la cual se inicia con el cuidado como núcleo central. Luego, desarrolla los metaparadigmas e indica dentro de estos el ambiente integral, incluye los aspectos del ambiente interior y exterior.

El interior incluye los sentimientos, significados y espiritualidad, a los que agrega los patrones del conocimiento y el personal, desarrollado a través del arte, meditación, danza, música, historias y otras expresiones aplicadas en la vida diaria y en la práctica de Enfermería, además del estético, que explora las experiencias y significados de vida y que incluye una presencia auténtica de enfermería como facilitador de cuidado. Luego, añade cuatro cuadrantes: en el superior izquierdo (YO), es el espacio del yo, es decir, subjetivo, se encuentra en el mundo interior de cada persona, incluye las emociones, recuerdos, percepciones, sensaciones inmediatas y estados de la mente (sueños, miedos, capacidad cognitiva, creencias y valores), mientras que el inferior izquierdo (NOSOTROS) es el espacio intersubjetivo que puede ser encontrado en el interior de la colectividad, es la manera conjunta de construir un bagaje cultural, historia y significados; disminuye la fragmentación y realza la práctica colaborativa y el diálogo profundo, brindando espacio al trabajo transdisciplinario y transpersonal.

Desde la mirada que plantea esta reflexión, los cuadrantes izquierdos describen aspectos de la realidad subjetiva, interpretativa y cualitativa, como el yo y nosotros, desde donde se justifica el uso de MCA fundamentada en esta teoría en trabajadores que perciben riesgos psicosociales, ya que las MCA permiten el crecimiento interior, potencian las características de las personas, de modo que aumenten el bienestar personal.

Ambas teóricas visualizan al ser humano desde una perspectiva integral con la que, en este caso, el trabajador puede abordar su salud o las alteraciones de producto de riesgos en el trabajo, motivo por el que se plantea, entonces, la posibilidad de desarrollar desde estas visiones un abordaje para las problemáticas psicosociales, desplegando saberes desde las terapias alternativas o complementarias. Como se observa, enfermería posee bases disciplinares que sustentan el cuidado de los trabajadores por medio de dichas intervenciones con el fin de que la profesión dote de un sustento científico a la salud laboral, mientras fortalece el bienestar de los trabajadores en forma holística y autónoma.

\section{CONCLUSIONES}

- Se visualiza un gran desafío para enfermería en el área de los riesgos psicosociales a nivel laboral, donde la participación de profesionales en enfermería en la consecución de este conocimiento es escasa aún, por lo que sugiere realizar levantamientos teóricos y empíricos desde el ámbito profesional y académico.

- En relación con las formas en que se aborda la temática hoy, se concluye que existen estrategias de abordajes teórico-prácticos para prevenir y tratar estas problemáticas a nivel laboral. Sin embargo, están basados en un paradigma positivista, el cual se enfoca en lo patológico, lo que complica el cuidado de los trabajadores con problemas psicosociales, motivo por el que se recomienda incorporar otros paradigmas en el cuidado para ofrecer alternativas de salud a los usuarios, según sus intereses y deseos. 
- Desde la enfermería, se puede colaborar en el enfrentamiento de los Riesgos psicosociales a nivel laboral. Para ello, se realizó una aproximación con dos visiones paradigmáticas de enfermería, cuyo fundamento son las bases teóricas de la disciplina y el abordaje de los riesgos psicosociales de manera holística e integral, los cuales potencian en el usuario el desarrollo de la mente, el cuerpo y el espíritu.

- Se postula que la medicina complementaria está siendo ampliamente utilizada por la sociedad actual, razón por la que la OMS se ha preocupado de regularizar la práctica respecto de estas temáticas, por ende, es de suma importancia que los profesionales de salud se instruyan en el tema y entreguen a los usuarios una nueva posibilidad en sus cuidados, en este caso, una nueva propuesta de abordaje para los riesgos psicosociales en el trabajo.

\section{Colaboradores y agradecimientos}

Las autoras trabajaron en conjunto todas las etapas de producción del manuscrito. Agradecen el apoyo del Plan de Mejoramiento Institucional Oncología UCM 1301, dependiente de la Universidad Católica del Maule, Talca, Chile, por su apoyo en la investigación desde donde emerge este documento; y al Centro del Cuidado, UC del Maule por la colaboración en la estructura de este documento.

\section{REFERENCIAS BIBLIOGRÁFICAS}

Cabieses, B., Miner, S. y Villegas, N. (2010). Análisis reflexivo del cuidado en reflexología y masoterapia centrado en la persona, por parte del profesional de enfermería. Cienc Enferm, 16 (1), 59 - 67

Centros de estudios de opinión ciudadana. (2010). Universidad de Talca. Diagnóstico situacional de medicinas complementarias alternativas en el país. [Acceso: 03/05/2012]. Recuperado de:

http://www.minsal.gob.cl/portal/url/item/9d59798ffb7dcbfae04001011f0139a0.pdf

Ceolin T., Heck, R., Pereira, D., Martins, A., Coimbra, C. y Silveira, D. (2009). Inserción de terapias complementarias en sistema único de salud atendiendo al cuidado integral de la asistencia. Enfermería global. [Acceso: 12/05/12], 16. Recuperado de: http://revistas.um.es/eglobal/article/view/66311

Dossey, B. (2008). Theory of Integral Nursing. Adv Nurs Sci, 31 (1), $52-73$

Dossey, B. y Keegan L. (2009). Holistic Philosophy, Theories and Ethics. En: Nursing: A Handbook for Practice. 5a ed. Canadá: Sudbury, M.A

Enfermeras holística. (2009-2015). Normativas históricas. [Acceso: 10/09/15]. Recuperado de: http://www.enfermeriaalternativa.cl/normativas_historico.html

Fawcett, J. (2005). Contemporary Nursing Knowledge. Analysis and Evaluation of Nursing Models and Theories. Philadelphia: F.A Davis Company. 
Gil-Monte, P. (2005). El síndrome de quemarse por el trabajo (burnout). Madrid: Ediciones Pirámide.

Gil-Monte, P. (2009). Algunas razonas para considerar los riesgos psicosociales en el trabajo y sus consecuencias en la salud pública. Rev Esp Salud Pública, 83,169-73.

Gobierno de Chile. (2012). Web Ministerio de Salud (MINSAL). Medicinas complementarias-Alternativas. [Acceso: 14/09/2015]. Recuperado de: http://web.minsal.cl/medicinas_complementarias

Hernández, T., Terán, O., Navarrete, D. y León, A. (2007). El síndrome de burnout: una aproximación a su conceptualización, antecedentes, modelos explicativos y de medición. Revista internacional la nueva gestión organizacional, 3(5), 50-68. [Acceso 10/09/15]. Recuperado de:

http://files.sld.cu/anestesiologia/files/2011/10/burnout-2.pdf

Kérouac, S., Pepin, J., y Ducharme, F., Duquette, A., y Major, F. (2002). El pensamiento enfermero. Madrid: Editorial Elvesier Masson.

Landsbergis, P. (2008). ¿Qué debe hacer para prevenir los riesgos psicosociales en el trabajo? Arch Prev Riesgos Labor, 11(1), 36-44

Martínez, S., y Hernández, A. (2005). Necesidad de estudios y legislación sobre factores psicosociales en el trabajo. Rev Cubana de Salud Pública, 31(4), 338 - 44.

Martínez, A. (2010). El síndrome de burnout, evolución conceptual y estado actual de la cuestión. Vivat Academia. [Acceso: 12/09/15]. 2010; 112. Recuperado de http://www.ucm.es/info/vivataca/numeros/n112/PDFs/Anbelacop.pdf

Mejía, M. (2008). Aplicación de algunas Teorías de Enfermería en la Práctica Clínica. Index Enferm, 17(3), 197200.

Meliá, J., Nogareda, C., Lahera, M., Duro, A., Peiró, J., Salanova, M. y García D. (2006). Principios comunes para la evaluación de riesgos psicosociales en la empresa. Barcelona.

Mitchell, G. (2007). La evolución humana. En: Modelos y teorías en enfermería. Marriner A, Raile M. $6^{\mathrm{a}}$ ed. Madrid: Elvesier.

Moreno, B. (2011). Factores y riesgos laborales psicosociales: conceptualización, historia y cambios actuales. Med. Segur. Trab. 2011, 57(1), 4 -19.

Navarro, Y. y Castro, M. (2010). Modelo de Dorothea Orem aplicado a un grupo comunitario a través del proceso de enfermería. Enfermería global. [Acceso: 13/09/15], 19. Recuperado de:

http://scielo.isciii.es/pdf/eg/n19/clinica3.pdf 
Nogareda, C., Gracias, E., Martínez, I. y Salanova, M. (2004). El trabajo emocional: concepto y prevención. Informe de investigación para el Ministerio de ciencia y tecnología. Universidad de Jaume I e Instituto Nacional de Seguridad e higiene en el trabajo.

Organización Internacional de trabajo/Organización Mundial de la Salud. (1984). Factores psicosociales en el trabajo: naturaleza, incidencia y prevención. [Acceso 16/09/2015]. Ginebra: OIT/OMS. Recuperado de: http://biblioteca.uces.edu.ar/MEDIA/EDOCS/FACTORES_Texto.pdf

Organización Mundial de la Salud. (2002). Estrategia de la OMS sobre medicina tradicional 2002-2005. OMS ediciones.

Panizzon, C., Luz, A. y Fensterseifer, L. (2008). Estresse da equipe de enfermagem de emergência clínica. Rev Gaúcha Enferm, 29(3), $391-9$.

Parse, R. (2014). The Humanbecoming Paradigm: A transformational Worldview. $2^{\circ}$ ed. Pittsburgh, Pennsylvania: A Discovery International Publication.

Peiró, J. (2004). El Sistema de Trabajo y sus implicaciones para la prevención de los riesgos psicosociales en el trabajo. Univ Psychol, 3(2), 179-86.

Pestka, E. Cutshall S. (2011). Integración de las terapias complementarias en la práctica de enfermería. En: Terapias complementarias y alternativas en enfermería. Traducción: $6^{\circ}$ ed. Complementary and alternative therapies in nursing. México D. F.: Manual moderno.

Quiceno J. y Vinaccia S. (2007). Burnout; "Síndrome de quemarse en el trabajo (SQT)". Acta colombiana de psicología, 10(2), $117-25$

Salanova, M., Llorens, S.(2008). Estado actual y retos futuros en el estudio del Burnout. Papeles del psicólogo. 29(1): $59-67$.

Secco, I., Robazzi, M., Souza, F. y Shimizu, D. (2010). Cargas psiquicas de trabalho e desgaste dos trabalhadores de enfermagem de hospital de ensino po Paraná, Brasil. Revista electrónica Saúde Mental Alcool e drogas. [Acceso: 14/09/15], 6(1): 1-15. Recuperado de: http://www.redalyc.org/pdf/803/80313414016.pdf

Snyder, M. y Lindquist, R. (2011). Terapias complementarias y alternativas en enfermería. Traducción: $6^{\circ}$ ed. Complementary and alternative therapies in nursing. México D. F.: Manual moderno.

Snyder, M., Niska K. y Lindsquit, R. (2011). Terapias complementarias y alternativas. Aspectos culturales de la atención. En Terapias complementarias y alternativas en enfermería. Traducción: $6^{\circ}$ ed. Complementary and alternative therapies in nursing. México D. F.: Manual moderno.

Sobrin, C. (2005). Las terapias naturales entran en escena en Enfermería. Excelencia en Enfermería. Revista Científica y de Divulgación, 12:12. 
Stacciarini, J. y Tróccoli, B. (2002). Estresse Ocupacional. En: Trabalho em Transicao, saúde em risco. Brasilia: Editora Universidade de Brasília.

Taub, M. y Olivares, V. (2009). Factores Psicosociales desde una perspectiva positiva. Ciencia y trabajo. 11(32): 27. 